\title{
Research Paper \\ The Effect of Eight Weeks Aquatic Therapy on Quality of Life, Movement Performance and Pain in Men With Fibromyalgia
}

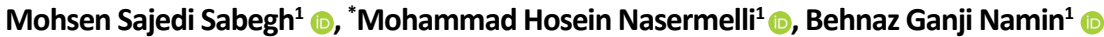

1. Department of Sport Injury and Corrective Exercises, Faculty of Physical Education \& Sports Sciences, Karaj Branch, Islamic Azad University, Karaj, Iran.

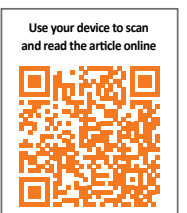

Citation Sajedi Sabegh M, Nasermelli MH, Ganji Namin B. The Effect of Eight Weeks Aquatic Therapy on Quality of Life, Movement Performance and Pain in Men With Fibromyalgia. Scientific Journal of Rehabilitation Medicine. 2021; 10(3):496-507. https:// doi.org/10.32598/sjrm.10.3.10

doi https://doi.org/10.32598/sjrm.10.3.10

Received: 11 Dec 2019

Accepted: 22 Jul 2020

Available Online: $23 \mathrm{Jul} 2021$

Keywords:

Aquatic training, Fibromyalgia, Pain, Quality of life, Function

\section{A B STRACT}

Background and Aims The purpose of the present study was to investigate the effect of a watercourse on the quality of life, pain, balance, and handgrip in men with fibromyalgia.

Methods The research method was quasi-experimental. The statistical population consists of men 40-50 years old with fibromyalgia in Tehran City. Among the available subjects who were purposefully selected, 30 were randomly divided into experimental and control groups (15 in each group). Quality of life was measured using the SF-36 questionnaire, perceived pain severity by visual grading scale, and strength using a manual dynamometer.

Results The results showed that 8 weeks of water exercise had a significant effect on the quality of life $(P=0.0001)$, pain $(P=0.0001)$, and strength gain in men with fibromyalgia $(P=0.0001)$.

Conclusion Water exercise can improve men's quality of life, strength, and pain perception with fibromyalgia.

\section{Extended Abstract}

\section{Introduction}

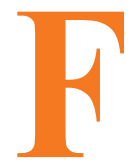

ibromyalgia is a common musculoskeletal disorder characterized by chronic diffuse musculoskeletal pain, dryness, paresthesia, drowsiness that does not rejuvenate, and premature fatigue, with numerous tactile points that are broadly and symmetrically extended. Are specified. Various causal mechanisms have been suggested for fibromyalgia to explain abnormal pain sensation. Many fibromyalgia patients referred to referral centers or rheumatologists show a high percentage of mental disorders.
This problem and the lack of a clear physiopathology have led some researchers to consider the origin of this disease as psychological. Preliminary studies of mental disorders in fibromyalgia patients, mainly through questionnaires filled out by the patients themselves, also showed a high prevalence of mental disorders. According to Goldberg, the percentage of people with fibromyalgia showed a history of major depressive disorder and the rate of a history of major depression. $16 \%$ of phobias were reported in patients with fibromyalgia. Also, these patients have more problems dealing with stress and being satisfied with their quality of life. Statistics show that the prevalence of chronic diffuse pain was $14 \%$ and $6 \%$, but the diagnosis of fibromyalgia was $4 \%$.

\section{${ }^{*}$ Corresponding Author:}

Mohammad Hosein Nasermelli, PhD.

Address: Department of Sport Injury and Corrective Exercises, Faculty of Physical Education \& Sports Sciences, Karaj Branch, Islamic Azad University, Karaj, Iran.

E-Mail: mh.naser.m@gmail.com 


\section{Methods}

Training in water due to its unique properties can be a very effective way of training, especially in pain and limited mobility conditions. In previous research, one of the variables has often been examined, while these affect each other. Reducing pain affects flexibility, as well as strength, and quality of life, and no research has been found that examines all of these at the same time. Therefore, it is necessary to conduct a study to investigate the effect of water exercises on fibromyalgia syndrome, and if this study is done, the results may lead to a suitable, easy and accessible treatment protocol to improve patients' symptoms.

This study aimed to investigate the effect of water exercise on quality of life, pain, and hand strength in men with fibromyalgia. The method of the present study was quasiexperimental. The statistical population included men aged 40-50 years with fibromyalgia in Tehran. Among the available subjects that were purposefully selected, 30 people were randomly divided into experimental training and control groups (15 people in each group).

Quality of life was measured using the SF-36 questionnaire, perceived pain intensity by a visual grading scale, and strength measured using a hand-held dynamometer. The results showed that 8 weeks of exercise in the water significantly affected men with fibromyalgia's quality of life, pain, and power. The inclusion criteria were as follows: the age range from 40-50 years old men with fibromyalgia diagnosed with a specialist was willing to participate in the study. The exclusion criteria were as follows: not suffering from acute and advanced orthopedic, psychological, diabetes, limb fractures, structural defects, and cardiovascular diseases that prevent the exercises considered in this study. Failure to use assistive devices such as crutches and walkers, avoidance of exercise or strenuous activity while participating in the survey, fear of water, and not participating in $50 \%$ of the exercises or three consecutive sessions were determined.

The research tools included a standard questionnaire of quality of life, visual analog scale test, performance evaluation including static and dynamic balance test, grip strength test with standard Yagami hand dynamometer, and flexibility assessment with sit and reach test. The training program included a training program taken from the sources approved by the referrals of the samples with warm-up stages, main exercises with progressive overload, and cooling for eight consecutive weeks with three training sessions per week with patients' conditions.
Two sections of descriptive statistics and inferential statistics have been used to analyze the information. Descriptive statistics have been used to classify and adjust the raw data to determine the main index of central tendency Mean \pm SD maximum and minimum of each variable and draw tables and graphs. A correlated t-test was used to evaluate the effect of training, and finally, an independent Ammon t-test was used to compare the experimental and control groups. All statistical calculations related to each of the hypotheses were performed using SPSS software, v. 21, at a significant level of $\mathrm{P}=0.05$.

\section{Results}

The results showed a significant effect of exercise in water on the quality of life. Due to the buoyancy and slight waves, the water environment disturbs the balance of people, in which case the person's balance is challenged, and a feeling of falling is created in him. This causes the person's body to try to prevent falling with balance strategies such as deep sense, strength, balance, etc.

\section{Discussion and Conclusion}

Our study showed improved quality of life, pain, strength, flexibility, and balance following a water exercise program in people with fibromyalgia. Therefore, with these exercises, people with fibromyalgia can improve their quality of life, which is one of the most important reasons for inactivity in these people. The second case was the positive effect of water exercise on motor performance (balance, strength, and flexibility). The pain was also reduced in these people, which was one of the most important reasons for improving these people's quality of life and motor function. Exercising in water relaxes the muscles and reduces the pressure on them. This can reduce the spasm, which ultimately reduces the pain of people with fibromyalgia. The positive effect of water exercises on flexibility was also observed. Joint flexibility and range of motion are related to joint mobility, muscle tone, the presence of destructive factors such as trigger points and spasms. Overall, the results showed that 8 weeks of exercise in water had a significant effect on quality of life, pain, range of motion and strength of men with fibromyalgia.

\section{Ethical Considerations}

\section{Compliance with ethical guidelines}

The ethical principles observed in the article, such as the informed consent of the participants, the confidentiality of information, the permission of the participants to cancel 
their participation in the research. Ethical approval was obtained from the Research Ethics Committee of the University of Medical Sciences/Karaj BRANCH research center (Ethical Code: IR.IAU.K.REC,1398.094).

Funding

This research did not receive any grant from funding agencies in the public, commercial, or non-profit sectors.

Authors' contributions

Authors contributed equally in preparing this article.

Conflict of interest

The authors declared no conflict of interest. 


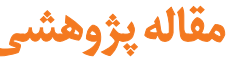

\section{ثأثير هشت هفته تمرين در آب بر كيفيت زندكى، عملكرد حركتى و درد مردان داراى فيبروميالزيا}

محسن ساجدى' هـ، "محمدحسين ناصرملى' ه، بهناز كنجى نمين' (1)

ا. كروه آسيبشناسى ورزشى و حركات اصلاحى، دانشكده تربيثبدنى و علوم ورزشى، واحد كرج، دانشعاه آزاد اسلامى، كرج، ايران.

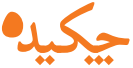

إنمينه و هدف هدف از تحقيق حاضر بررسى تأثير يك دوره تمرين در آبه بر كيفيت زندكى، درد و قدرت كرفتن دست مردان داراي فيبروميالزيابود.

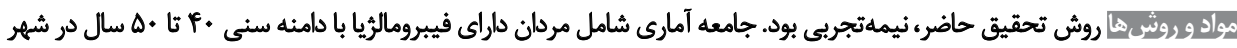

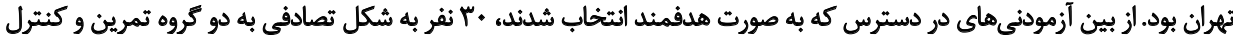

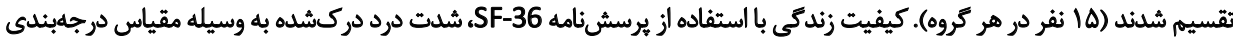

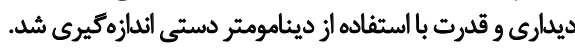

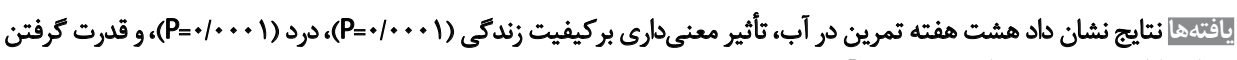

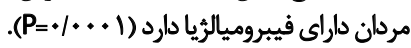

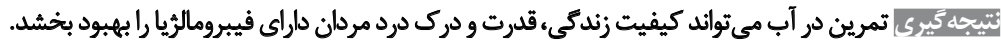

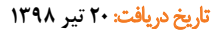

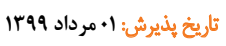

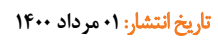

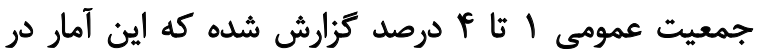

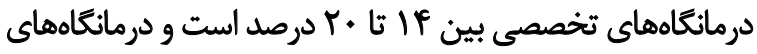

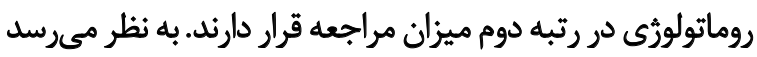

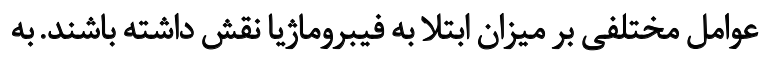

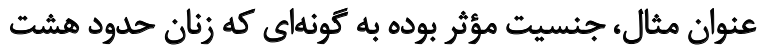

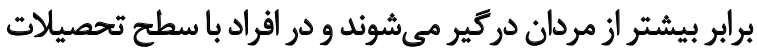

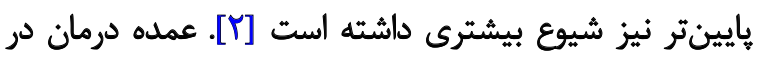

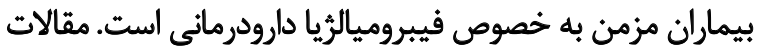

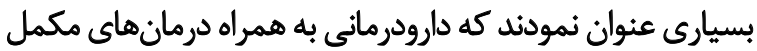

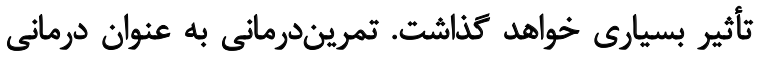

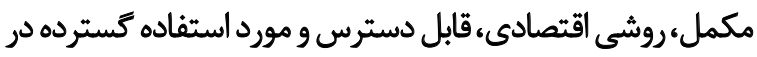

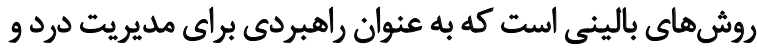

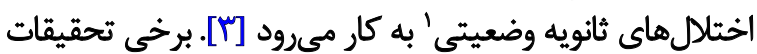

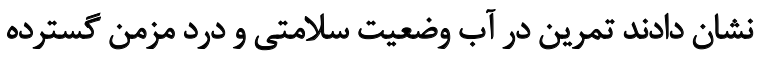

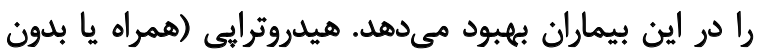

\section{Secondary position}

مelat

فيبروميالريا يك اختلال شايع عضلاتي اسكلتي است كه با درد

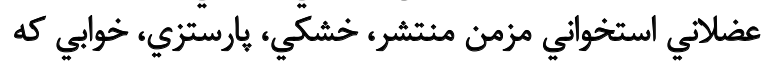

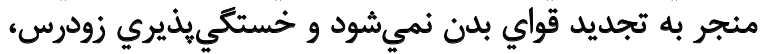

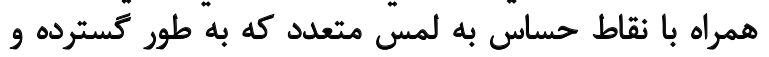

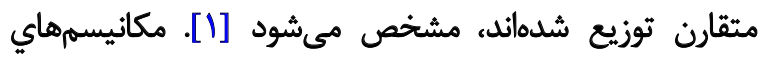

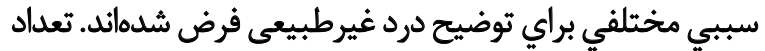

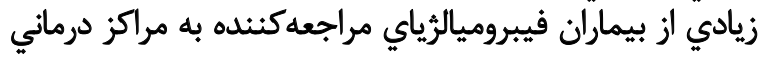

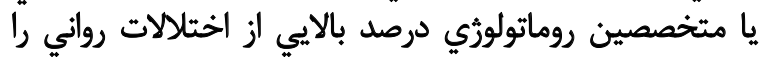

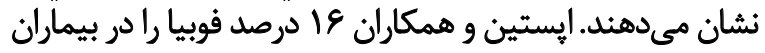

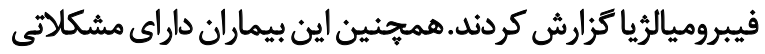

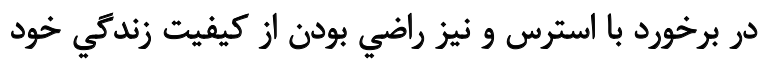

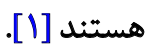

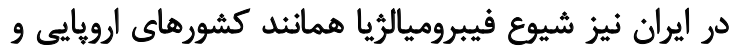

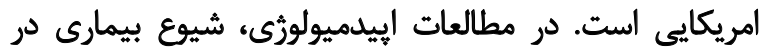


تحقيق مىشد، استفاده از وسايل كمكى نظير عصا و واكر، ترس

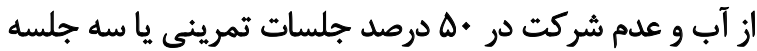

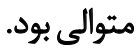

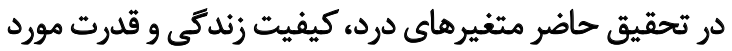

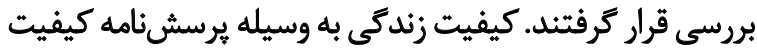

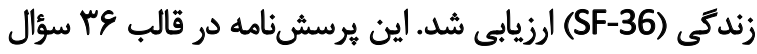

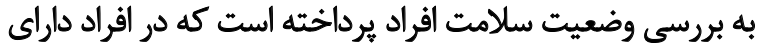

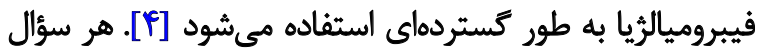

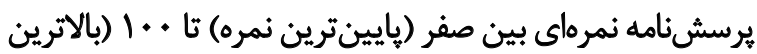

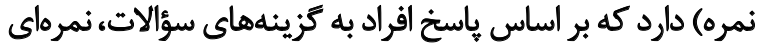

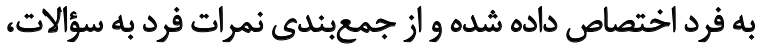

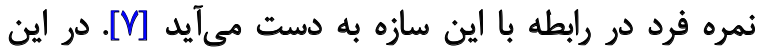

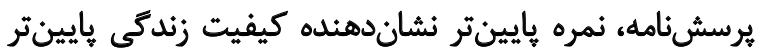

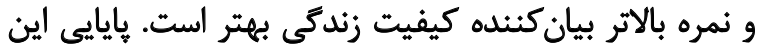

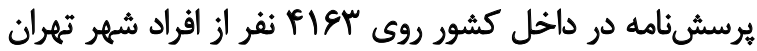

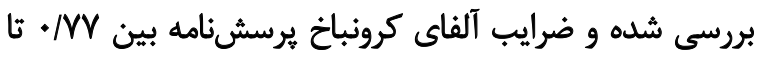

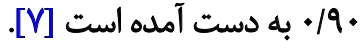

به منظور اندازمكيرى شدت درد ادراكشده، از مقياس

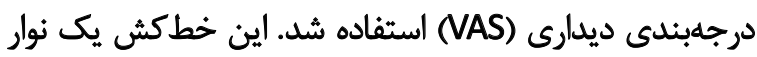

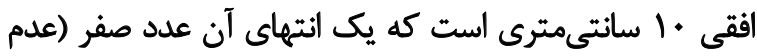

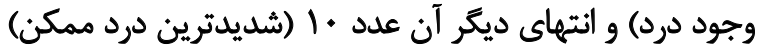

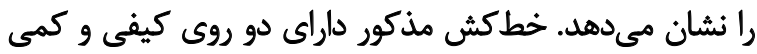

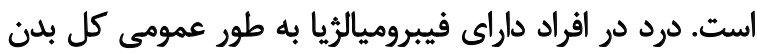

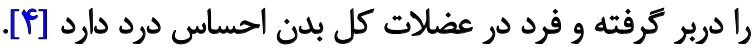

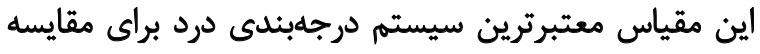

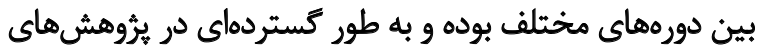

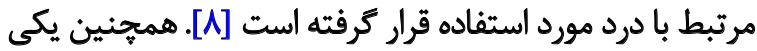

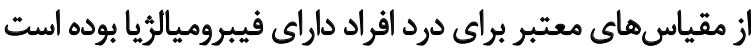

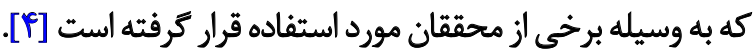

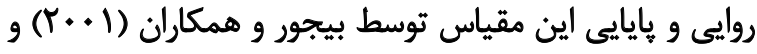

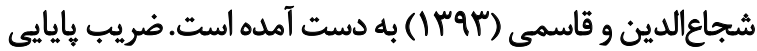

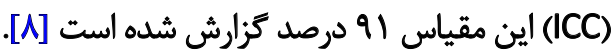

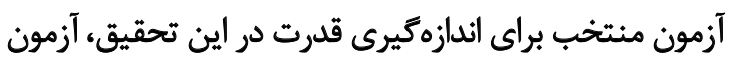

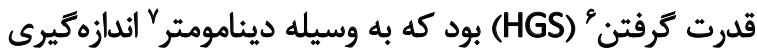

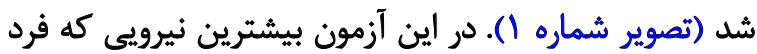

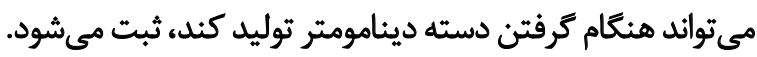

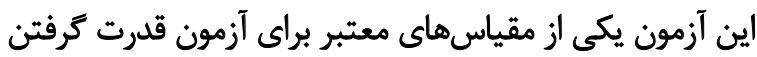

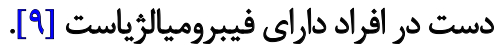

در تحقيق حاضر جهت طراحى تمرينات ورزشى، از برنامه

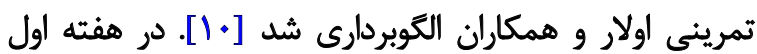
تمرينات، حداكثر تعداد تكرارى كه آزمودنى (تا حد خستيكى)

6. Handgrip Strength (HGS) test

7. Dynamometer
تمرين) به خاطر خواص فيزيكى آب (مانند گرما و غوطهورى)

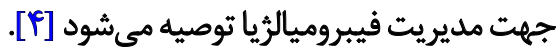

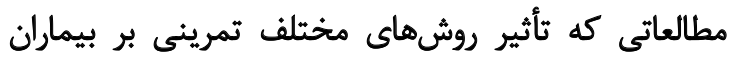

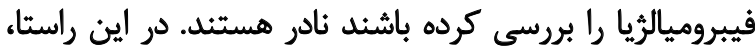

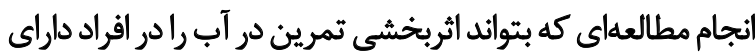

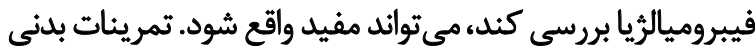

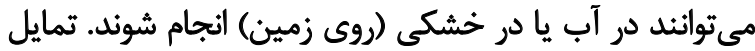

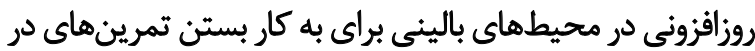

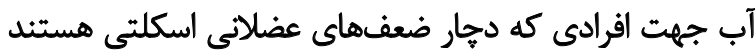

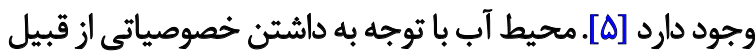

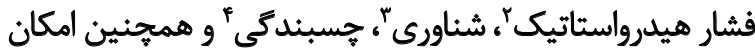

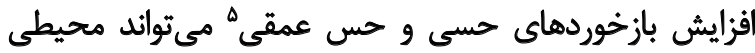

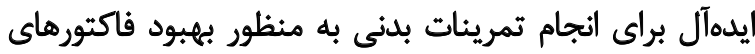

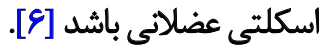

در تحقيقات كذشته اغلب يكى از متغيرهاى درد، كيفيت

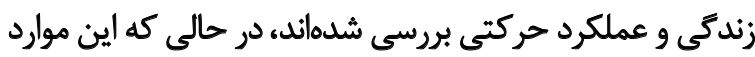

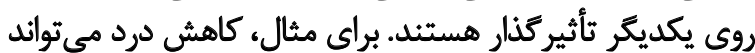

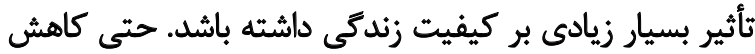

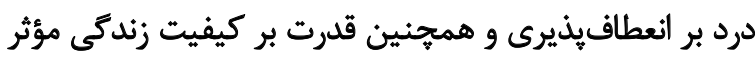

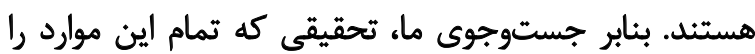

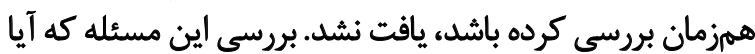

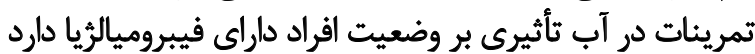

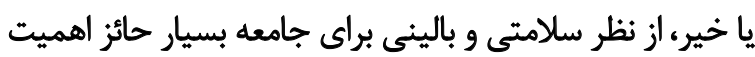

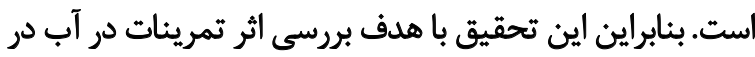

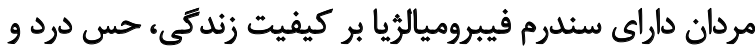
قدرت ترفتن دست انجام شد.

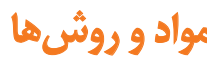

در اين مطالعه از روش نيمهتجربى استفاده شد و طرح تحقيق

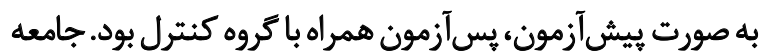

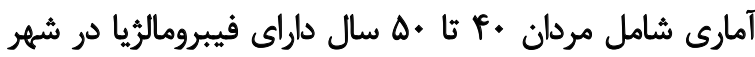

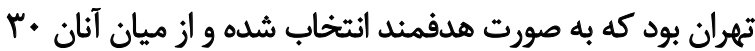

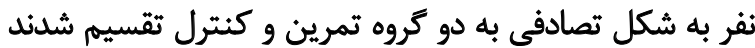

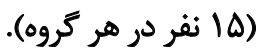

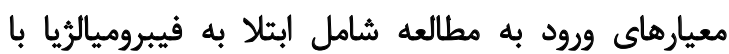

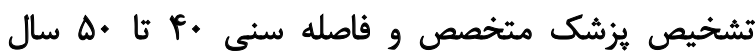

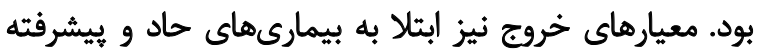

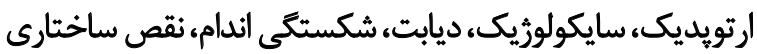

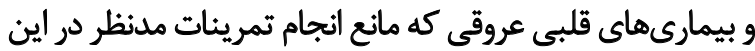

2. Hydrostatic pressure

3. Floating

4. Water adhesion

5. Proprioception 
جدول ا. برنامه تمرينى به مدت هشت هفته

\begin{tabular}{|c|c|c|c|c|c|c|c|c|c|}
\hline & & & مر هر جلسه & تعداد تكرار & & & & & \\
\hline هفته & هفته & هفته & هقته & هفته & هغته & هفته & هفته & تمرين & 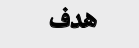 \\
\hline هشتم & هفتم & ششم & ينجم & جهارم & 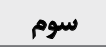 & دوم & اول اول & & \\
\hline- & - & - & - & - & - & - & - & يباددروى ب دقيقه & \\
\hline - & - & - & - & - & - & - & - & كشش عضلات بايينتنه & كرم كردن هنا. \\
\hline- & - & - & - & - & - & - & - & كشش عضلات بالاتنه & \\
\hline PXTYMT & AXY $-M$ & $P X Y=-1 A$ & $|x| \varepsilon-11$ & Pxle-18 & PXIY-If & $|x| \cdot-\mid 1 Y$ & $P \times A-1$. & خمه كردن و باز كردن ران در وضعيت تكيه به بهان & \\
\hline RXYMTM & PXY.MY & $P X Y \cdot-11$ & $|x| y-11$ & Pxle-1e & fXIY-If & $|x|=-1 Y$ & $P \times A-1$. & 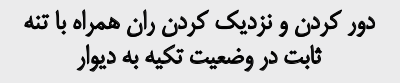 & \\
\hline PXYYTE & $R X Y=-T^{\prime}$ & $P X Y=-1 A$ & $|x| y-11$ & $|x| f-18$ & $f \times \mid r-18$ & $F(x)=-14$ & $1 \times 1-1$. & فلكثن و اكستنشن بازو در وضعيت تكيه & تمرينات \\
\hline RXYYMP & AXY.MY & $P X Y=-11$ & $|x| \varepsilon-11$ & Pxle-1E & PXIY-If & $|x|=-1 Y$ & $P \times A-1$. & دور شلن و تزديك شلن بازو در وضعيت تكيه & 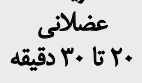 \\
\hline PXYYME & RXY.-MY & $F X Y=-1 A$ & $P x \mid y-11$ & $|x| f-18$ & $F X \mid Y-18$ & $|x| \cdot-11$ & $P \times 1-1$. & فلكشن اقفى و اكستنشن افقى بازو & \\
\hline RXYT-ME & RXY.-MY & $P X Y=-1 A$ & $|x| y-11$ & $\mid f \times 1 f-18$ & $f x \mid y-18$ & $r \times 1+-14$ & $1 \times 1-1$. & خم كردن همزمان ران، زانو و ميج با در & \\
\hline RXYM-TE & 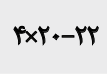 & FXY. $-1 A$ & $|x| 9-11$ & $|x| f-18$ & $F X \mid Y-I f$ & $|x|+-11$ & $F \times A-1$. & 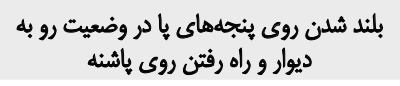 & \\
\hline- & - & - & - & - & - & - & - & بياده روى ب دقيقه & بازكشت به \\
\hline - & - & - & - & - & - & - & - & كشش بايينتثه & 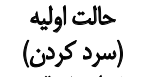 \\
\hline - & - & - & - & - & - & - & - & كشش بالاتنه & ه تا ما دقيقه \\
\hline
\end{tabular}

و مَروه كنترل از آمون تى مستقل استفاده شد. كليه محاسبات

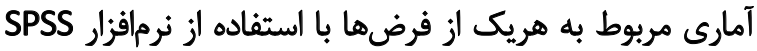

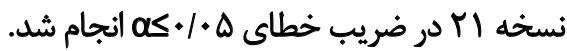

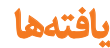

برخى از ويزگكىهاى جمعيتشناختى آزمودنىها در جدول

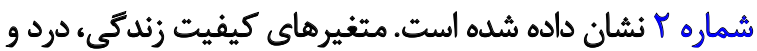

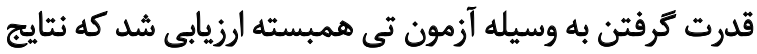

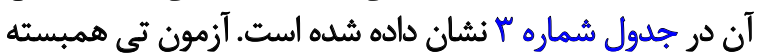

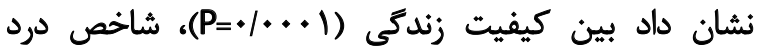

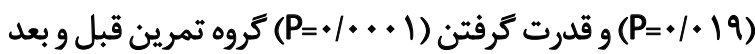

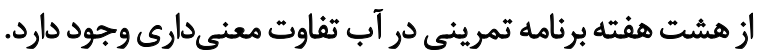

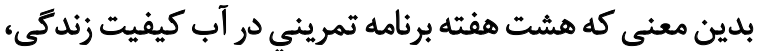

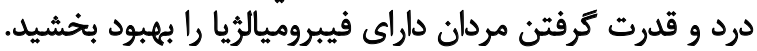

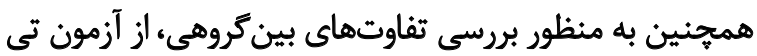

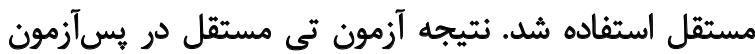

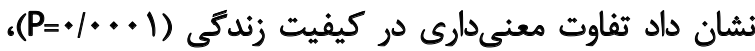

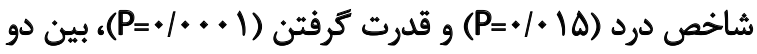
كروه تمرين وكنترل وجود دارد (جدول شماره (f) (f).
مى توانست انجام دهد اندازهميرى شد و ميانكين آنها بـ به عنوان

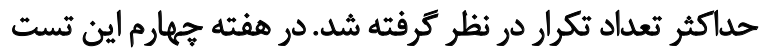

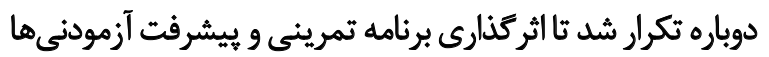

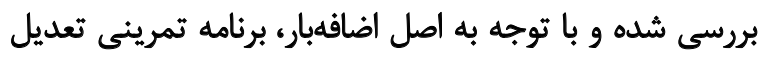

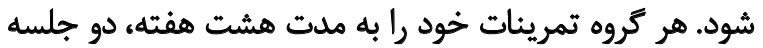

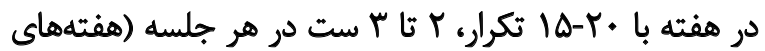

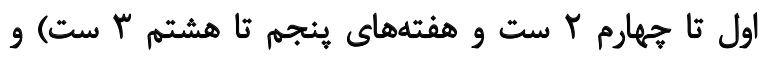

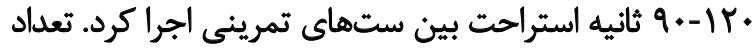

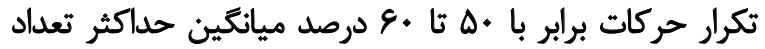

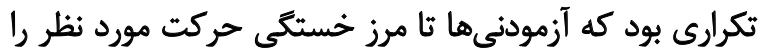

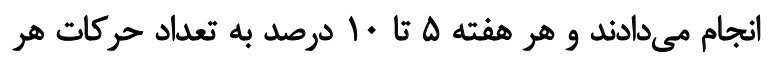

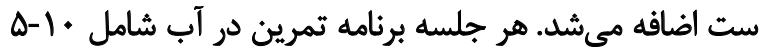

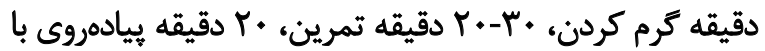

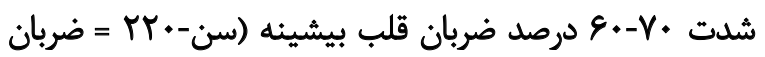

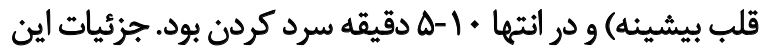

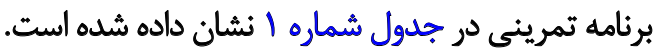
در اين يرؤشش جهت تجزيه و تحليل اطلاعات از دو بخش آمار

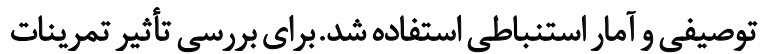
از آزمون تى همبسته و درنهايت به منظور مقايسه كروه تمرين ترين تريناتين 


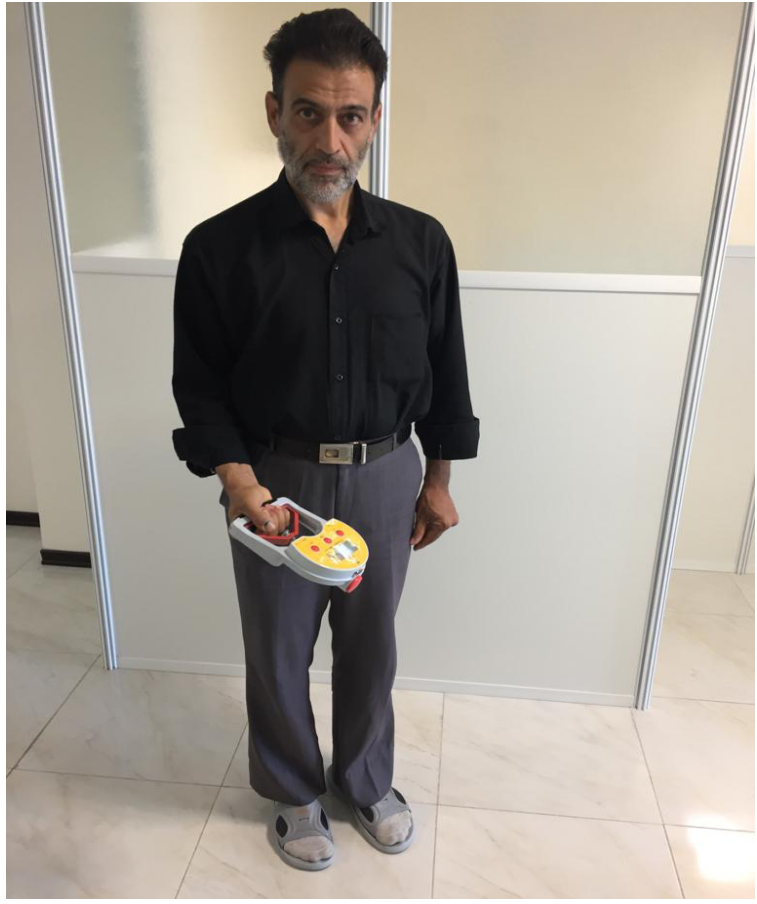

طبتروانبخشي

تصوير ا. نحوه أنجام آزمون قدرت كرفتن دست

[10] و ليونن و همكاران [19] همراستا بوده كه عنوان كردند

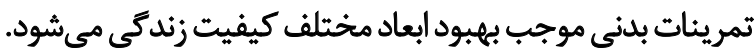

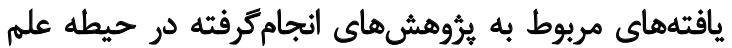

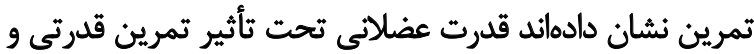

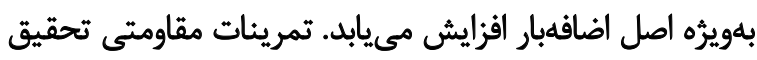

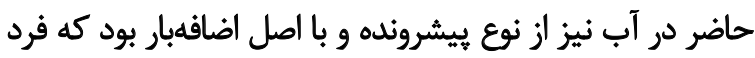

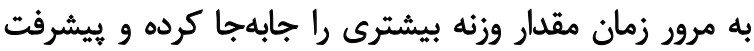

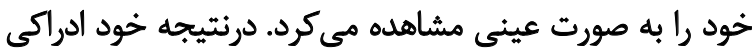

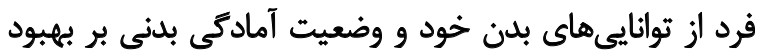

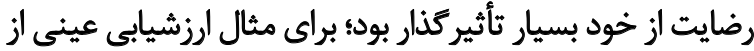

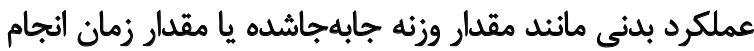

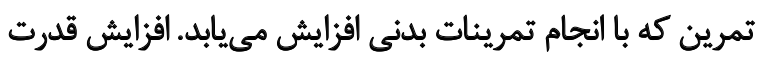

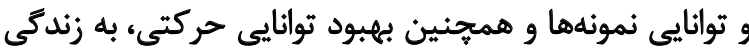

\section{بحث}

تحقيق حاضر نشان داد تمرين در آب مىتواند باعث بهببود

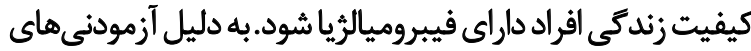

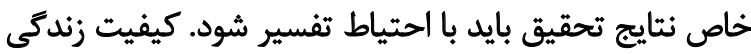

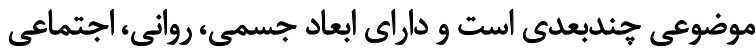

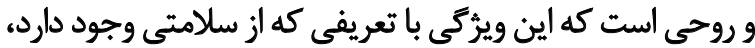

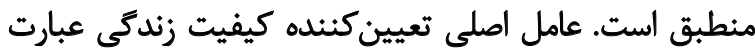

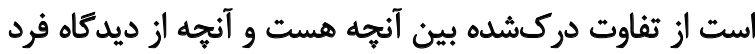

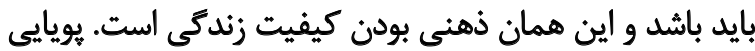

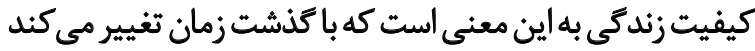

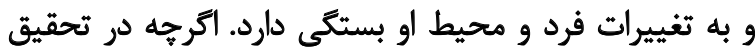

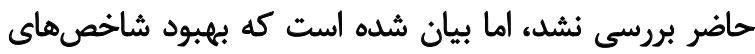

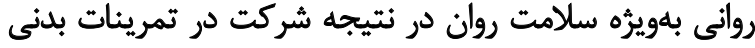

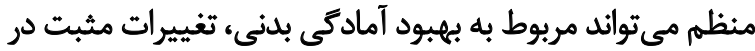

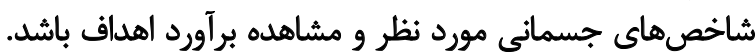

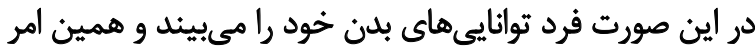

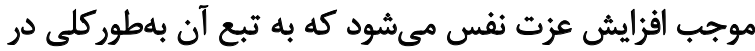

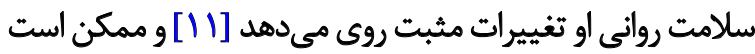

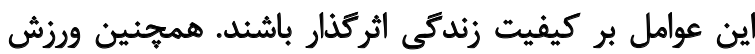

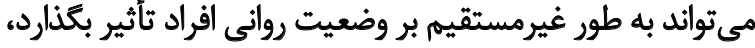

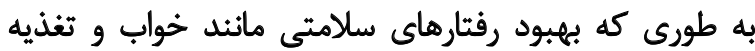

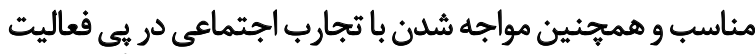

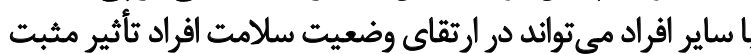

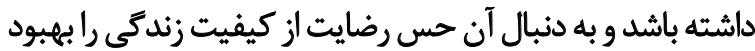

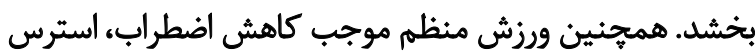

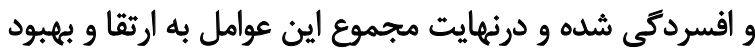

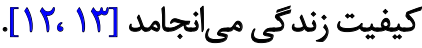

اكثر تحقيقاتى كه كيفيت زندكى راني را بررسى كردهاند،

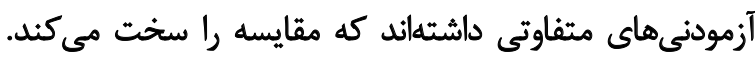

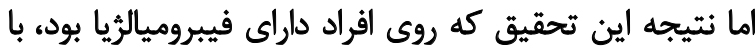

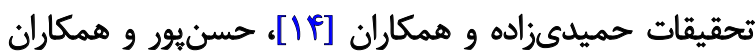

جدول r. ويرُكى هاى جمعيتشناختى نمونها (ها نفر در هر كروه)

\begin{tabular}{|c|c|c|}
\hline ميانكين+انحرافمعيار & تمروه & متغيرها \\
\hline$\Delta T / T+ \pm F / T$ & تمرين در آب & \multirow{2}{*}{ سن (سال) } \\
\hline$\Delta r / \circ \pm E / R$ & كنترل & \\
\hline $\mid V / / \Delta \cdot \pm r / \Lambda \Delta$ & تمرين در آب & \multirow{2}{*}{ قد (بانتىمتر) } \\
\hline $\mid V Q / . . \pm \psi / a \varphi$ & كتترل & \\
\hline$W / A+ \pm V / \Delta r$ & تمرين در آب & \multirow{2}{*}{ وزن (كيلوكرم) } \\
\hline$V Q / q, \pm r / K r$ & كتثرل & \\
\hline
\end{tabular}


جدول ب. نتيجه آزمون تى همبسته براي بررسى تأثير برنامه تمرينى در آب بر كيفيت زئدكى، درد و قدرت كرفتن دست مردان داراى فيبروميالزيا (كروه تمرين 10 نفر)

\begin{tabular}{|c|c|c|c|c|c|}
\hline سطح معنى دارى & $\mathbf{t}$ & df & مياتكيند|نحرافمعيار & زمان & مثغير \\
\hline $.100+1$ & $A$ & if & $\begin{array}{l}\forall \notin Y Y \\
\Delta Y \pm \& / V\end{array}$ & يبي آزّهون & كيفيث زندكى \\
\hline .1 .19 & $r$ & if & $\begin{array}{l}\Delta \pm 1 \\
\pm \pm 1\end{array}$ & يبش يس أزمون & شاخص درد \\
\hline .1 .0 .1 & 9 & if & $\begin{array}{l}M \pm 1 \\
r \pm 1\end{array}$ & يسيش آزمون & قدرت كرقتن \\
\hline
\end{tabular}

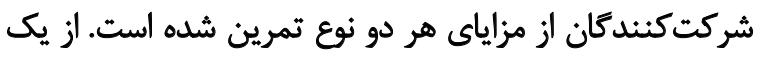

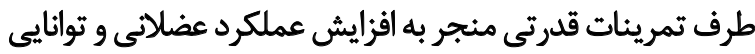

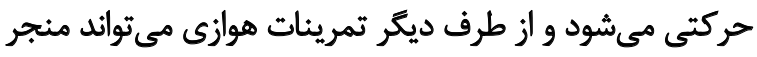

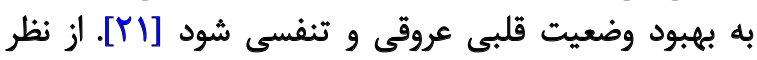

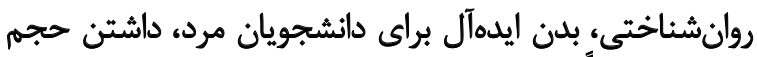

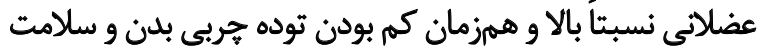
عمومى قلبى تنفسى است.

ورزش باعث افزايش جريان خون به مغز مي شئود و دستكاه

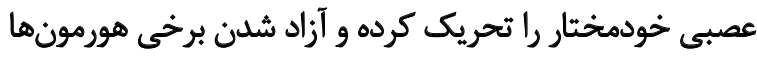

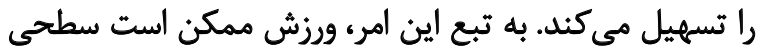

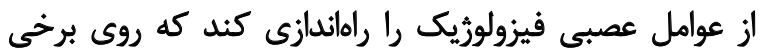

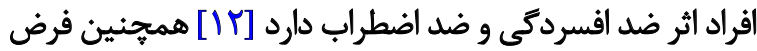

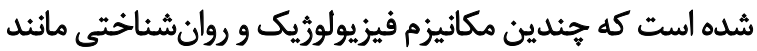

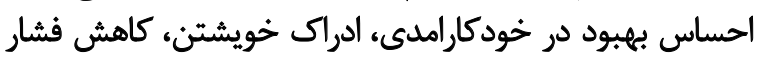

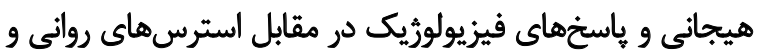

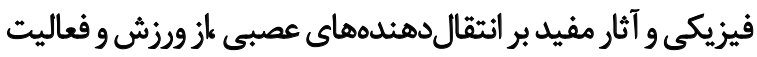

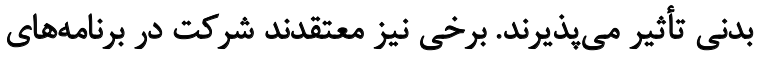

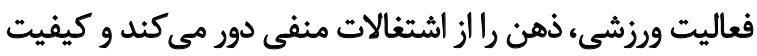

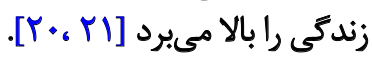

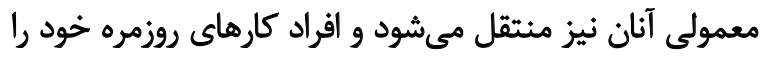

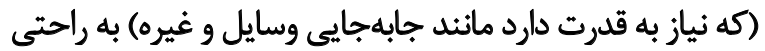

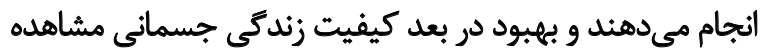
مىشود [IV] كاهش وزن و تناسب اندام يكى از مهمثرين دلايل شركت در

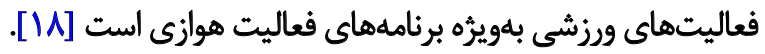

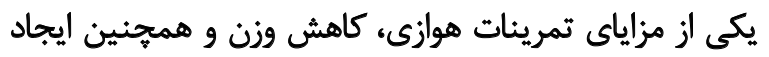

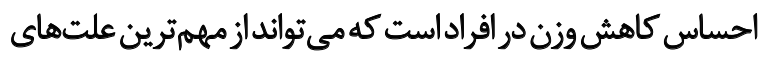

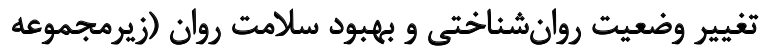

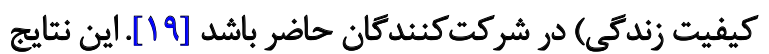

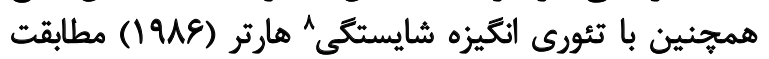

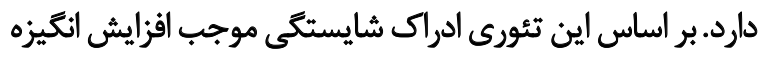

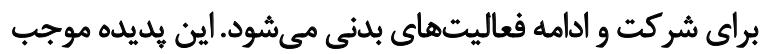

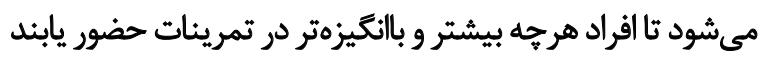

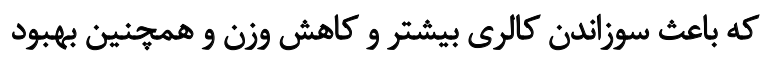

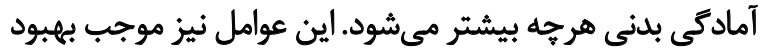

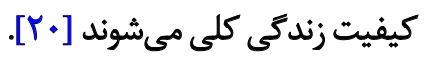
به احتمال زياد تركيب اين تمرينات منجر به بهرهمندى

جدول f. نتايج آزمون تى مستقل براي مقايسه كيفيت زندكى، درد و قدرت كرفتن دست در يسآزمون دو كروه (تمرين و كثترل)

\begin{tabular}{|c|c|c|c|c|c|}
\hline سطح معنى دارى & df & $\mathbf{t}$ & مياتكيندانحرافمعيار & تمووه & متغير \\
\hline. $\mid+\ldots 1$ & M & $H / \cdot V$ & $\begin{array}{l}\Delta Y \pm S N \\
P A \pm Y\end{array}$ & كتمرين & كيفيت زندكى \\
\hline $.1 \cdot 10$ & ru & $r$ & $\begin{array}{l}r \pm r \\
\Delta \pm 1\end{array}$ & كتمرين & شاخص درد \\
\hline$+1+\ldots 1$ & 队 & 1 & $\begin{array}{l}r \pm 1 \\
r \cdot \pm r\end{array}$ & تمرين & قدرت كرفتن \\
\hline
\end{tabular}




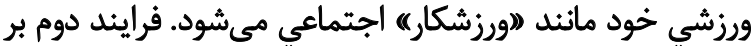

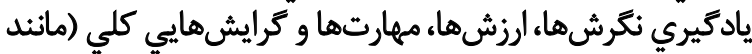

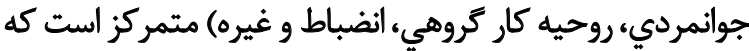

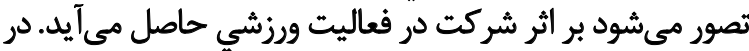

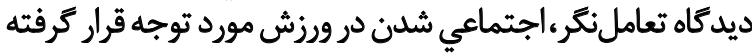

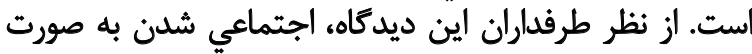

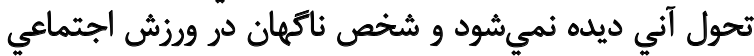

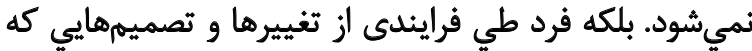

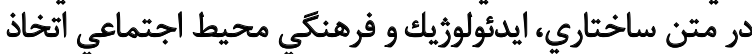

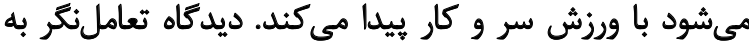

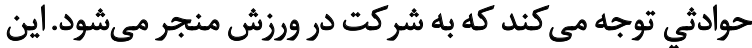

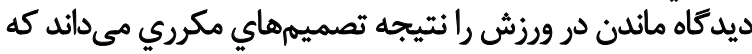

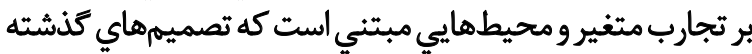

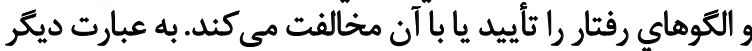

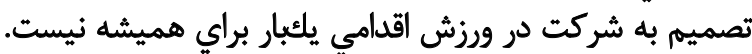

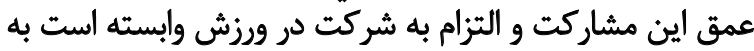

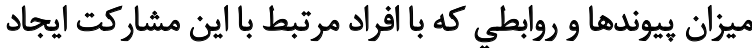

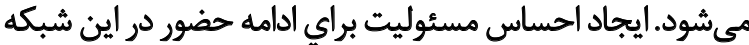

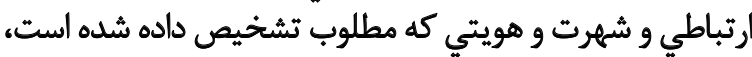

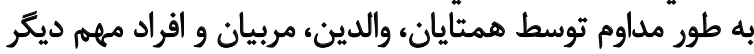

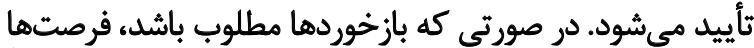

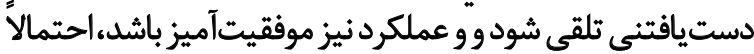

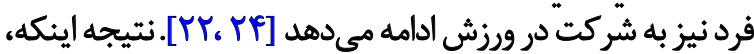

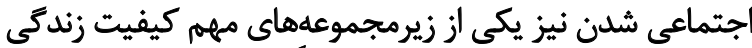

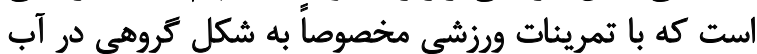

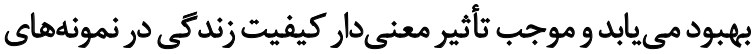
داراي فيبروميالريا در تحقيق حاضر شده استي ميتي

با توجه به اينكه مهمثرين مؤلفه برنامه مداخله تمرينى در

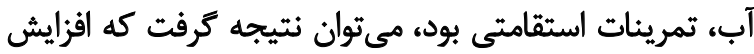

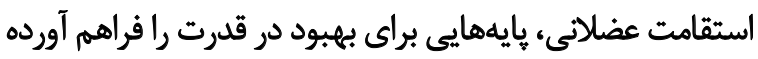

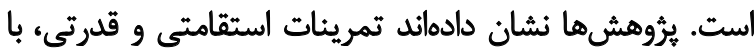

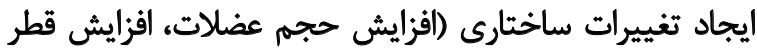

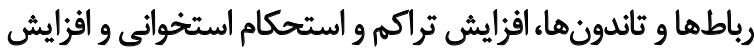

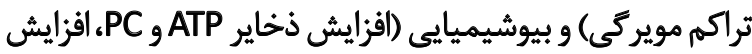

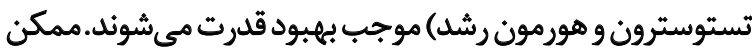

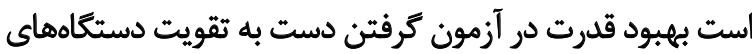

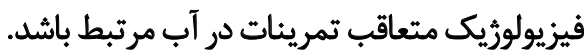

خاصيت شناورى در آب باعث مىشود بيماران آزادانهتر و

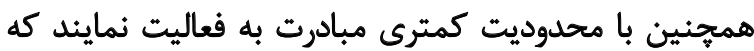

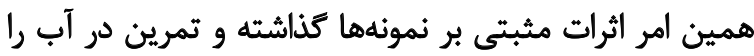

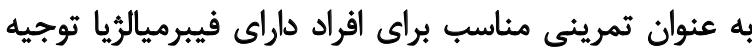

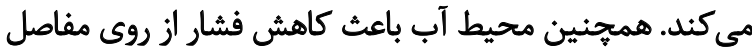

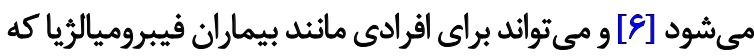

افراد داراى فيبروميالريا در بسيارى موارد از عملكرد جسماني

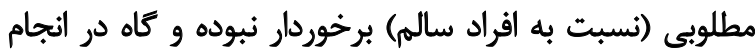

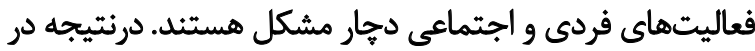

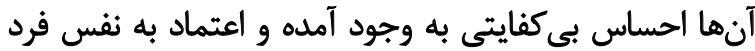

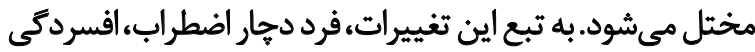

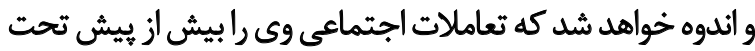

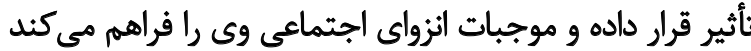

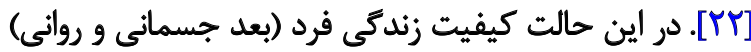

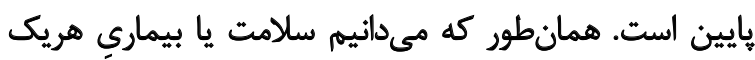

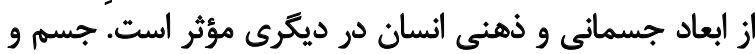

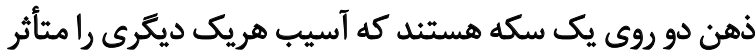

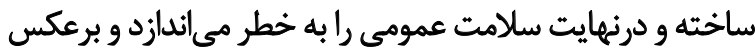

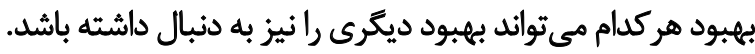

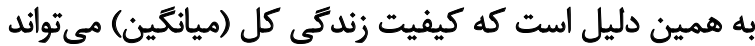
مثأثر از تمرينات در آب باشد.

بورك و همكاران (ع + (Y) در تحقيقى به بررسى نوع فعاليت

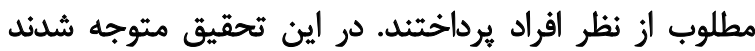

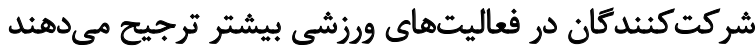

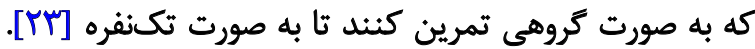

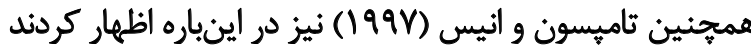

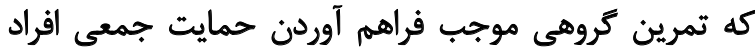

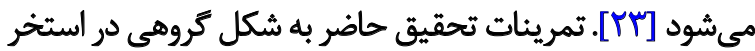

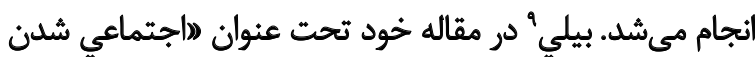

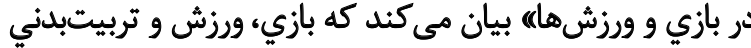

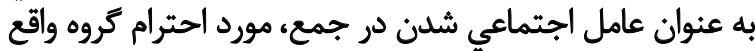

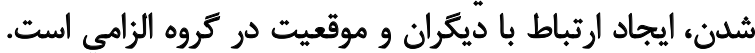

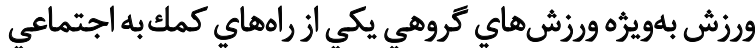

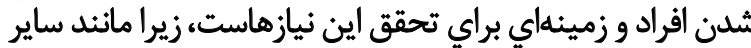

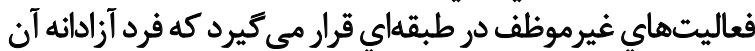

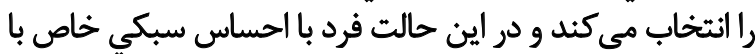

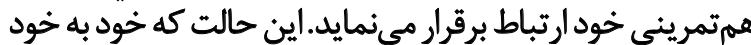

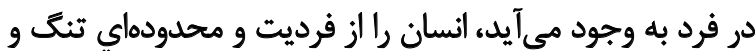

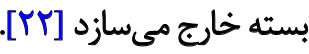

اغلب مطالعات اجتماعي شدن درورزش حول دو مسئله متمركز

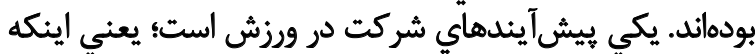

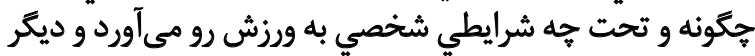

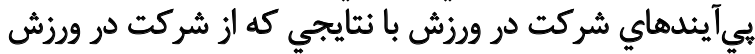

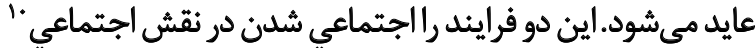

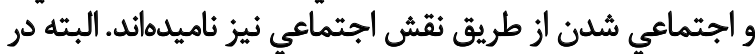

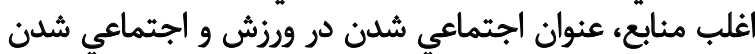
زز طريق ورزش به جشم مي خورد. در فرايند اول، فرد در نقش وري 


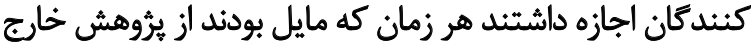

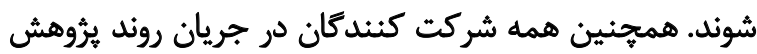
بودند. اطلاعات آن ها محرمانه نكَه داشته شد شدان درين

اين تحقيق هيج كونه كمك مالى از سازمان هاى تأمين مالى در بخش هاى عمومى، تجارى يا غيرانتفاعى دريافت نكردي

$$
\text { مشاركت نويسندكًان }
$$

تمام نويسندكان در آمادهسازى اين مقاله مشاركت يكسان

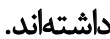

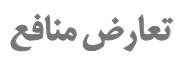

بنابر اظهار نويسندكان، اين مقاله تعارض منافع ندارد.
مشكلات حركتى در خشكى دارند كه باعث درد مىشود، كزينه

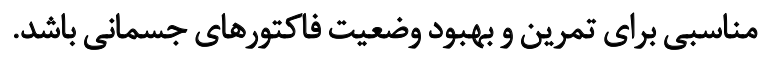

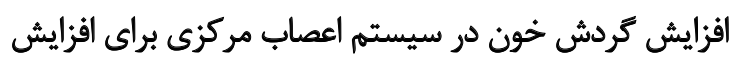

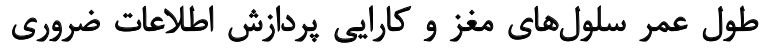

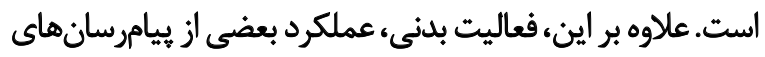

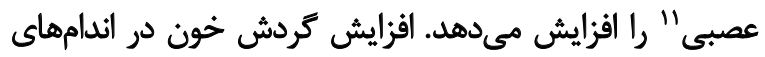

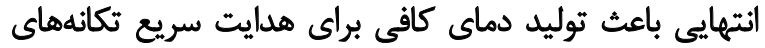

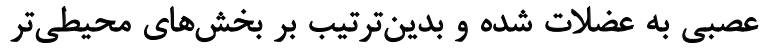

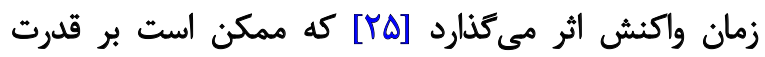
آزمودنى ها مؤثر بوده باشد.

تمرين در آب موجب ريلكس شدن عضلات و كاهش فشار

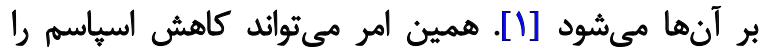

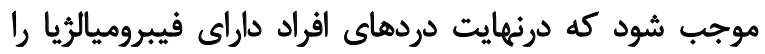

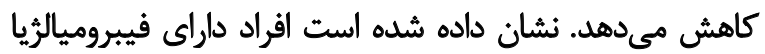

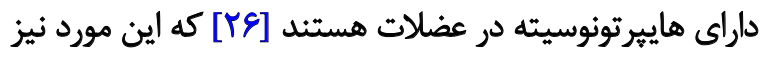

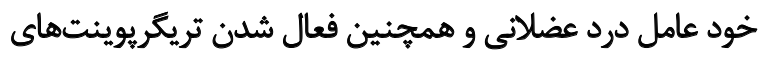

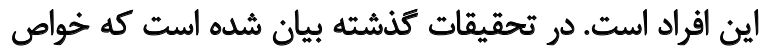

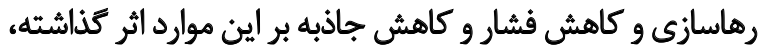

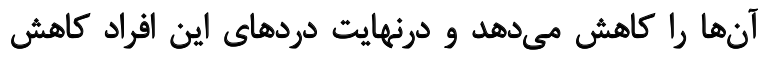

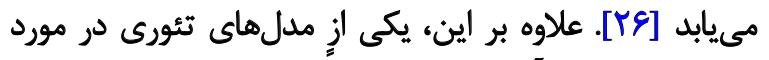

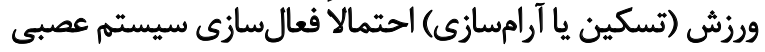

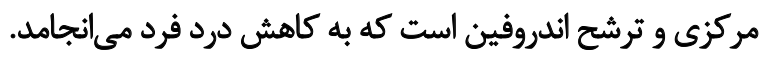

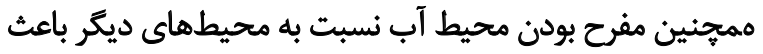

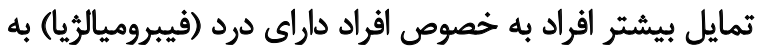

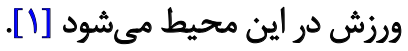

\section{نتئنه}

نتايج تحقيق ما حاكى از بهبود كيفيت زندگىى، درد و قدرت

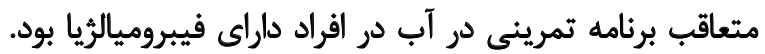

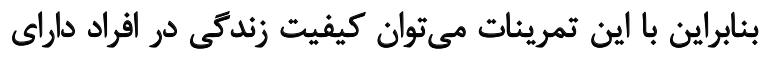

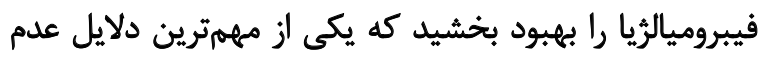

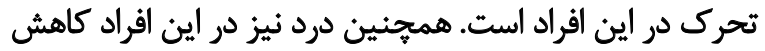

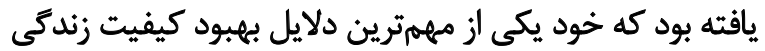

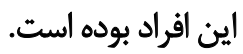

مالاحظان أخاقي

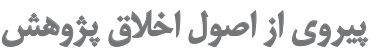

در اجراى يرؤهش ملاحظات اخلاقى مطابق با دستورالعمل

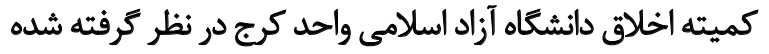

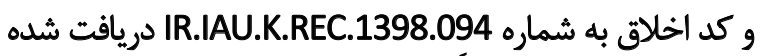
است. اصول اخلاقى تماماً در اين مقاله رعايت شده است. شركت 


\section{References}

[1] Neira SR, Marques AP, Pérez IP, Cervantes RF, Costa JV. Effectiveness of aquatic therapy vs land-based therapy for balance and pain in women with fibromyalgia: A study protocol for a randomised controlled trial. BMC Musculoskeletal Disorders. 2017; 18(1):22. [DOI:10.1186/s12891-016-1364-5] [PMID] [PMCID]

[2] Khatibi Aghda A, Hali Saz M, Asheghan M, Shams al-Dini A, Sobhani V, Labaf S. [Quality oflife of fibromyalgia patients referred to Baqiyatallah hospital physical medicine and rehabilitation clinic (Persian)]. Veterinary Medicine. 2012; 6(21):20-6. https://www. sid.ir/fa/journal/ViewPaper.aspx?ID=214875

[3] Aparicio VA, Ortega FB, Heredia JM, Carbonell-Baeza A, Sjöström $M$, Delgado-Fernandez $M$. Handgrip strength test as a complementary tool in the assessment of fibromyalgia severity in women. Archives of Physical Medicine and Rehabilitation. 2011; 92(1):83-8. https://www.sciencedirect.com/science/article/abs/ pii/S0003999310007732

[4] Sevimli D, Kozanoglu E, Guzel R, Doganay A. The effects of aquatic, isometric strength-stretching and aerobic exercise on physical and psychological parameters of female patients with fibromyalgia syndrome. Journal of physical therapy science. 2015; 27(6):1781-6. [DOI:10.1589/jpts.27.1781] [PMID] [PMCID]

[5] Devereux K, Robertson D, Briffa NK. Effects of a water-based program on women 65 years and over: A randomised controlled trial. Australian Journal of Physiotherapy. 2005; 51(2):102-8. [DOI:10.1016/S0004-9514(05)70038-6]

[6] Douris P, Southard V, Varga C, Schauss W, Gennaro C, Reiss A. The effect of land and aquatic exercise on balance scores in older adults. Journal of Geriatric Physical Therapy. 2003; 26(1):3-6. [DOI:10.1519/00139143-200304000-00001]

[7] Borzoo S, Arastoo A, Ghasemzade R, Zahednezhad S, Habibi A, Latifi SM. [Effects of aerobic exercise on quality of life in residents of geriatric homes, Ahvaz, Iran (Persian)]. Salmand: Iranian Journal of Ageing. 2011; 6(1):47-51. http://salmandj.uswr.ac.ir/ article-1-376-fa.html

[8] Shojaedin S, Ghasemi F. [The effect of two methods of exercise therapy on chronic low back pain in military personnel (Persian)]. Journal of Military Medicine. 2014; 16(1):1-7. http://militarymedj.ir/article-1-1189-fa.html

[9] Aparicio VA, Ortega FB, Heredia JM, Carbonell-Baeza A, Sjöström $M$, Delgado-Fernandez $M$. Handgrip strength test as a complementary tool in the assessment of fibromyalgia severity in women. Archives of Physical Medicine and Rehabilitation. 2011; 92(1):83-8. https://www.sciencedirect.com/science/article/abs/ pii/\$0003999310007732

[10] Avelar NC, Bastone AC, Alcântara MA, Gomes WF. Effectiveness of aquatic and non-aquatic lower limb muscle endurance training in the static and dynamic balance of elderly people. Brazilian Journal of Physical Therapy. 2010; 14:229-36. [DOI:10.1590/ S1413-35552010000300007]

[11] Seyed Ahmadi M, Keyvanlou F, Koushan M, Mohamadi Raof M. [Comparison of mental health status in athlete and non-athlete students (Persian)]. Journal of Sabzevar University of Medical Sciences. 2010; 17(2):116-22. https://www.sid.ir/fa/journal/ViewPaper.aspx?id $=125948$
[12] Straub OS. Health Psychology. New York: Worth Publisher; 2002. https://psycnet.apa.org/record/2002-17430-000

[13] Nilsen TS, Raastad T, Skovlund E, Courneya KS, Langberg CW, Lilleby W, et al. Effects of strength training on body composition, physical functioning, and quality of life in prostate cancer patients during androgen deprivation therapy. Acta oncologica. 2015; 54(10):1805-13. [DOI:10.3109/028418 6X.2015.1037008] [PMID]

[14] Hamidizade S, Ahmadi F, Aslani Y, Etemadifar SH, Salehi K, Kord Yazdi R. [The effect of an exercise program on the healthquality of life in older adults (Persian)]. Journal of Shahid Sadoughi University of Medical sciences. 2007; 16(1):81-6. http:// jssu.ssu.ac.ir/article-1-553-en.html

[15] Hassanpour Dehkordi A, Masudi R, Naderipoor A, Poor mir Reza Kalhori R. [The effect of an exercise program on the health quality of life in older adults (Persian)]. Salmand: Iranian Journal Of Aging. 2006; 2(6):437-44. http://salmandj.uswr. ac.ir/article-1-58-fa.htm

[16] Leinonen R, Heikkinen E, Hirvensalo M, Lintunen T, Rasinaho $M$, Sakari-Rantala $R$, et al. Customer-oriented counseling for physical activity in older people: study protocol and selected baseline results of a randomized-controlled trial (ISRCTN 07330512). Scandinavian Journal of Medicine \& Science in Sports. 2007; 17(2):156-64. [DOI:10.1111/j.16000838.2006.00536.x] [PMID]

[17] Zambom-Ferraresi F, Cebollero P, Gorostiaga EM, Hernández $\mathrm{M}$, Hueto J, Cascante J, et al. Effects of combined resistance and endurance training versus resistance training alone on strength, exercise capacity, and quality of life in patients with COPD. Journal of Cardiopulmonary Rehabilitation and Prevention. 2015; 35(6):446-53. [DOI:10.1097/ HCR.0000000000000132] [PMID]

[18] Campbell A, Hausenblas HA. Effects of exercise interventions on body image: A meta-analysis. Journal of Health Psychology. 2009; 14(6):780-93. [DOI:10.1177/1359105309338977]

[19] Crossen K, Raymore LA. Body Attitudes and participation in physical activity, are they related for adolescent females? New Zealand Journal of Sports Medicine. 1997; 25:42-5.

[20] Morgan WP, Goldston SE. Exercise and mental health. United Kingdom: Taylor \& Francis; 2013. [DOI:10.4324/9780203780749]

[21] Assessing Levels of Physical Activity. The ALPHA Health Related Fitness Test Battery for children and adolescents [Internet]. 2010 [Updated 2010]. Available from: http://www.ugr. es/ cts262/ES/documents/ALPHA-FitnessTestManualforChildren-Adolescents.pdf

[22] Parry KD, Jones I, Wann DL. An examination of sport fandom in the United Kingdom: A comparative analysis of fan behaviors, socialization processes, and team identification. Journal of Sport Behavior. 2014; 37(3):251-67. [DOI:10.1177/1012690210380582]

[23] Burke SM, Carron AV, Eys MA. Physical activity context: Preferences of university students. Psychology of Sport and Exercise. 2006; 7(1):1-3. [DOI:10.1016/j.psychsport.2005.03.002] 
[24] Khademi A. [Comparison of psychosocial characteristics of athletic and non-athletic college students (Persian)] [MSc. Thesis]. Guilan: University of Guilan; 2006. https://ganj.irandoc.ac.ir/\#/articles/03e3218f617809382222b93c7ffa6f27

[25] Kamijo K, Nishihira Y, Hatta A, Kaneda T, Wasaka T, Kida T, et al. Differential influences of exercise intensity on information processing in the central nervous system. European Journal of Applied Physiology. 2004; 92(3):305-11. [DOI:10.1007/ s00421-004-1097-2] [PMID]

[26] Martin DG, Wiener CD, de Freitas CX, Costa JL, Rombaldi AJ, Oses JP. Levels of brain-derived neurotrophic factor in patients with fibromyalgia and chronic low back pain: Results of an aquatic physical therapy protocol. FACETS. 2018; 3(1):12-21. [DOI:10.1139/facets-2017-0016] 